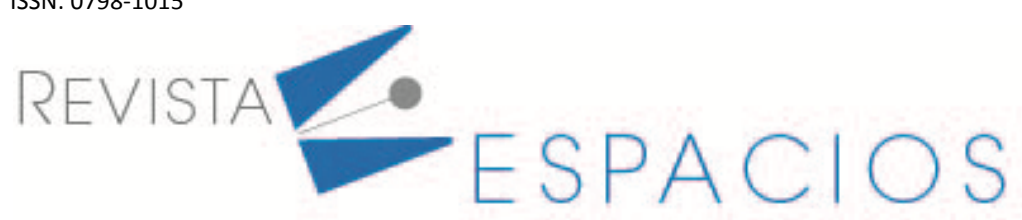

\title{
Violencia y dependencia emocional en parejas adolescentes de educación secundaria de la región Huancavelica, Perú
}

\author{
Violence and emotional dependence in high school adolescent couples in Huancavelica \\ region
}

\author{
GONZALES-CASTRO, Abel ${ }^{1}$ \\ GUERRA-OLIVARES, Tula Susana² \\ RODRIGUEZ-BENITES, Carlos ${ }^{3}$
}

\begin{abstract}
Resumen
La violencia y dependencia emocional en parejas adolescentes de educación secundaria está presente en todos los estratos sociales. El objetivo fue establecer la relación entre violencia y dependencia emocional en estudiantes mujeres con pareja de educación secundaria. Los resultados reflejan que un tercio de la muestra sufrió violencia y dependencia emocional moderado y severo, debido al predominio del machismo, desconocimiento de derechos de las mujeres adolescentes y carencia de habilidades emocionales en adolescentes de la región.
\end{abstract}

Palabras clave: violencia en parejas adolescentes, dependencia emocional, violencia y dependencia.

\begin{abstract}
Violence and emotional dependence in high school adolescent couples is present in all social strata. The objective was to establish the relationship between violence and emotional dependence in female students with a high school partner. The results reflect that a third of the sample suffered moderate and severe violence and emotional dependence, due to the prevalence of machismo, ignorance of the rights of adolescent women, and lack of emotional skills in adolescents in the region.

Key words: teen dating violence, emotional dependence, violence and dependence.
\end{abstract}

\section{Introducción}

La violencia en parejas adolescentes de educación secundaria es un problema que está presente en la historia de la sociedad y no tiene fronteras. Se evidencia tanto en países pobres, como emergentes y del primer mundo; este último grupo con altos ingresos como América del Norte, países de Europa Central, Occidental y Oriental (Gracia, 2014), incluido el Reino Unido. La Organización de las Naciones Unidas (2014) informó que más de un millón de mujeres fueron víctimas de violencia, por lo que se debe considerar como una cuestión de urgencia internacional. Leen et al. (2013) expresan que, durante el noviazgo, jóvenes de la comunidad afroamericana también muestran violencia como efecto del racismo, bajo nivel socio económico y crecimiento de vecindarios

\footnotetext{
${ }^{1}$ Docente e investigador. Universidad Nacional de Huancavelica. Perú. Contacto: abel.gonzales@unh.edu.pe Orcid: https://orcid.org/0000-0001-72513452

2 Docente e investigador. Universidad Nacional de Huancavelica. Perú. Contacto: tula.guerra@unh.edu.pe Orcid: https://orcid.org/0000-0002-4583-6384

${ }^{3}$ Docente e investigador. Universidad Nacional de Huancavelica. Perú. Contacto: carlos.benites@unh.edu.pe Orcid: https://orcid.org/0000-0001-94376364
} 
pobres (Racine y Zeytinoglu, 2012); del mismo modo, los estudios de Vagi et al. (2015) indican que muy pocos estudiantes no sufrieron ningún tipo de violencia, quedando evidente que las mujeres, en un mayor porcentaje, son víctimas de los agresores. Sin embargo, es necesario precisar que tanto varones como mujeres pueden ser agresores o receptores. En el caso de México, en los adolescentes predomina la agresión física y sexual (ZamoraDamián et al., 2018).

Con el desarrollo de las tecnología de información y comunicación (TIC) y las redes sociales, los dispositivos electrónicos son utilizados como instrumentos de agresión contra las parejas adolescentes, situación que se extiende por todo el mundo. García y Cerda (2012) precisan que, en los países nórdicos con modelo económico de bienestar, la violencia digital, psicológica, sexual y física está presente en $42,9 \%$. En la parte occidental de África, Nigeria, donde la población mayoritaria es musulmana, también se tiene información de la presencia de violencia física, psicológica, emocional, sexual por novios, padres, vecinos y parientes (Erulkar, 2001), lo mismo sucede en los jóvenes judíos y árabes en Israel durante el noviazgo, donde los hombres muestran una tasa mayor de agresividad (Sherer, 2009).

En España, los resultados de una encuesta nacional indican que la prevalencia de violencia íntima afecta a las mujer de todos los estratos sociales en $25 \%$, sin embargo, varía la frecuencia y la categoría según la posición socioeconómica Ruiz-pérez et al., 2017. Por otra parte, la (Organización Mundial de la Salud, 2013b) describe la prevalencia de la violencia contra las mujeres por sus parejas en más de un tercio en Asia sudoriental $(37,7 \%)$, Mediterráneo oriental (37\%), África (36,6\%). Del mismo modo, en una encuesta de estudio comparativo en 12 países de América Latina y el Caribe a adolescentes y mujeres, realizada por García-Moreno, Guedes y Knerr (2013, pp.2-3), para la Organización Panamericana de la Salud (OPS) y la OMS se encontró que: "la mayoría de las mujeres (61\% a 93\%) informaron haber sufrido violencia física de su pareja en los últimos 12 meses también informaron haber padecido violencia emocional". OMS, 2017 publicó que en todo el mundo casi un tercio (30\%) de las mujeres han sufrido alguna forma de violencia física o sexual de parte de su pareja en algún momento de su vida. Cienfuegos (2014), en Padilla y Cruz (2019), acota: muchas mujeres que tienen una relación de pareja pueden estar experimentado un estado de violencia sin saberlo. En la región donde se realizó la investigación, el fenómeno de la violencia puede ser generado por varios factores, siendo algunos, el predominio de la cultura machista, el qué dirán por la familia y la sociedad, obligando a mantener la información oculta.

La violencia en parejas adolescentes también es considerada como un problema de salud pública, que quebranta los derechos humanos, causa efectos en la salud mental, física, sexual y emocional siendo los más frecuentes: depresión, alcoholismo, infecciones de transmisión sexual, embarazos no deseados, abortos, nacimiento de bebés con bajo peso, lesiones y muertes (OMS, 2017). Del mismo modo, estos conflictos y tragedias ocasionan dolor, también pérdidas económicas en las familias nucleares y familias extendidas, afectando mucho más a los hogares pobres y de extrema pobreza, así como al Estado por los gastos administrativos en las instituciones públicas como el Ministerio Público, Poder Judicial, Centro de Salud, entre otros.

Las causas de violencia identificadas en las parejas de sexo femenino según la (OMS, 2003) responden a: trastornos de la personalidad, depresión, haber presenciado o sufrido violencia en la niñez, dominio masculino en la familia (machismo), familias disfuncionales, sanciones débiles contra la violencia en la comunidad, bajo grado de instrucción, pobreza, normas sociales que fomentan la violencia y el consumo de alcohol; de la misma manera, los programas televisivos como novelas, peliculas cinematográficos con imágenes violentas contra la mujer, que son pésimos modelos que imitan muchos adolescentes ejecutores como receptores de violencia; la existencia de patrones socioculturales que reproducen relaciones desiguales de poder y las diferencias jerárquicas de hombres sobre mujeres, influyen en el incremento de la violencia, según explicaciones de la Defensoría del Pueblo (2018). Estos patrones son actitudes, creencias imaginarias, prejuicios presentes en la población, que justifican y toleran la violencia hacia las parejas adolescentes y adultas. 
Una vez que inicia la violencia en una relación de pareja sea de adolescentes o adultos, es poco probable que se detenga con el transcurso del tiempo, por lo que existe una necesidad de formar, educar a los adolescentes a respetar y hacer respetar sus derechos. Al respecto García y Cerda, 2012 precisan qué si desde el noviazgo existe violencia hacia la pareja, es muy alta la probabilidad de que persista y sea mucho más severa en el matrimonio o en la unión libre.

Villegas y Sanchez (2013) afirman que las víctimas pueden sentir la imposibilidad de salir de la relación de maltrato ya que están sujetas a ella, sea por la fuerza física, la dependencia emocional, por el aislamiento social, vínculos económicos, legales o sociales; también otros aspectos psicológicos como el abandono o el desaliento, la presión familiar o social, la falta de alternativas de vida, un futuro desamparado al cual se verán enfrentados. Estas consideraciones descritas son evaluadas por las mujeres como dificultades significativas para salir adelante por sí solas.

Frente a este problema los estados miembros de la Organización de las Naciones Unidas (ONU), en el año 2000 y en septiembre del 2015, declararon los Objetivos de Desarrollo del Milenio para reducir la pobreza extrema, siendo uno de los objetivos la igualdad de género, con plazo al 2015 y luego al 2030. Sin embargo, a la fecha las mujeres y niñas siguen sufriendo discriminación y la violencia, se hace más resistente en todas partes del mundo (PNUD, 2000; PNUD, 2015; OMS, 2015), porque la violencia es cultural, se manifiesta en la educación, ciencia, religión, arte, leyes, ideología, y las organizaciones, incluyendo las instituciones educativas "donde las prácticas de violencia no se presentan solo entre niños y jóvenes, sino también en los actores institucionales, como docentes, directivos, personal administrativo y demás colaboradores... La violencia ha permeado todas las relaciones humanas" (Ospina-Ramírez y Ñañez, 2019 p.205).

En esta investigación se consideró la definición de Roque (2015) quién manifiesta que la pareja adolescente es la relación amorosa sostenida entre dos personas con o sin intención de casarse, asimismo Rodríguez y Kjeizer (2002), citado en Roque (2015), puntualizan que: “Es una relación social explícitamente acordada entre dos personas para acompañarse en las actividades recreativas y sociales, y en la cual se expresan sentimientos amorosos y emocionales a través de la palabra y los contactos corporales" (p. 87).

El equilibrio de una gestión emocional saludable en las parejas de estudiantes de educación secundaria se da con la práctica del amor sano o deseo de estar con el otro con libertad, que sería la primera forma de relación. Una segunda forma: dependientes emocionales pasivos, adictos a la evitación o relaciones con total ausencia de sentimientos (egoísta y con tendencia al narcicismo), siendo un extremo; con características: a) evaden intensamente la relación, al dedicarse con pasión a otras actividades fuera de la relación sentimental (suele tratarse de adicciones), b) evitan la relación con objeto de protegerse a sí mismos de la absorción y control por parte de la otra persona, c) evitan el contacto íntimo con su pareja, para lo cual utilizan una variedad de técnicas de distanciamiento. La tercera forma: los dependientes emocionales pasivos, se sienten atraídos por sujetos distantes, poco accesibles, difíciles, fríos, poco afectivos, con el premisa: "no me conviene, pero no puedo perderlo", con las siguientes características: a) dedican una cantidad desproporcionada de tiempo, una atención y un valor por encima de sí mismos, a la persona a la que son adictas, y esa forma de centrar la atención tiene a menudo una cualidad obsesiva. b) tienen expectativas irreales por lo que se refiere a la consideración positiva incondicional de la otra persona que forma parte de la relación, c) descuidan la atención que deben proporcionarse o valorarse a sí mismos mientras están en la relación con la otra persona (Mellody en Castelló, 2005).

La dependencia emocional aborda un vínculo afectivo entre el dependiente emocional dominante y el pasivo, que son los que generan los desequilibrios en la relación de parejas adolescentes (Castelló, 2005), es decir, las parejas con esos tipos de dependencia carecen de habilidades para una gestión saludable de las emociones o una vida emocional saludable. La causa fundamental para que un adolescente adquiera ese comportamiento, 
según Castelló (2005), es la necesidad emocional insatisfecha de atención de los padres durante la infancia, asi como la vivencia en ambientes familiares de violencia. Pérez, Momeñe, Olave, Estévez, y Iruarrizaga (2019) afirman que el abuso infantil, los hogares disfuncionales y la pobreza, generan otras causas secundarias como la baja autoestima, miedo a la soledad y el estado de ánimo negativo que induce a la dependencia emocional.

Bosch (2009), explica que los adolescentes que tuvieron un apego seguro con el amor de sus padres, tienen mayor capacidad de mantener una relación de amor sosegada, cálida y gratificante; tendrán menores problemas durante el tiempo que dure la unión de pareja, no tienen temor al abandono, tienen la misma confianza que tuvieron de sus padres, practican una relación de pareja horizontal; totalmente contrario, en los niños con carencias de amor, maltratados, abusados, y con padres autoritarios, estos en la adolescencia no estarán seguros que los amen de verdad, bucarán afectos en parejas con el modelo de amor que recibieron en la infancia, relacionándose muchas veces con parejas de sentimiento evitativo y dominante (frio, distante, esquivo, con miedo a amar, e incomodo en un contexto afectuoso y expresivo) con el argumento: "para que te voy a amar, si tú me vas a dejar". La dependencia emocional pasiva se manifiesta durante la adolescencia y a inicios de la vida adulta (Castelló, 2005).

La dependencia emocional definida como necesidad profunda de afecto, contacto continuo y excesivo por parte de la pareja (Momeñe et al., 2017) con mucho temor al abandono (Vallejo, 2019) y (Sophia et al., 2007) generando un intercambio de sentimientos asimétricos, situando al otro miembro de la pareja, su ídolo, en el centro de su existencia; así en adelante, corre el riesgo de mantener una relación malsana, unilateral, de malos tratos, vejaciones, desprecios de su pareja; sin embargo (Izquierdo y Gómez- Acosta, 2013), el dependiente pasivo, lucha por mantener la relación por miedo a perder la pareja por su baja autoestima, negándose afecto así mismos, fomentando en su par un comportamiento dominante. Castelló (2013) afirma que "La dependencia emocional se define como un patrón persistente de necesidades emocionales insatisfechas que se intentan cubrir desadaptativamente con otras personas" (p. 17), donde lo más relevante para el dependiente emocional es el mundo del amor, si pierde, el duelo es inmenso e insoportable, en comparación a una persona dependiente normal es tres veces más intensa, siendo un duelo patológico, que requiere ayuda. Castelló (2005) enuncia que la vida de los dependientes emocionales será un calvario que puede llegar a límites extremos, según el carácter de su pareja, (...) que propicien la aparición de violencia psíquica y física. Si la persona tiene una dependencia emocional grave, aceptará agresiones, humillaciones, infidelidades continuas, burlas, menosprecios, con tal de no romper su relación de pareja.

Vallejo (2019), asegura que estas personas presentan: baja autoestima, obsesión por la otra persona, perciben un sentido distorsionado de la realidad, son fáciles de ser controladas y manipuladas porque no tienen confianza en sí mismas, lo cual afecta en su desempeño académico, laboral, relaciones familiares, sociales y básicamente la salud mental, a pesar que dicen sentirse felices e imprescindibles apoyando al que "necesita ayuda".

Los adolescentes dependientes emocionales (Martín y De la Villa, 2019) son personas vulnerables, se eclipsan fácilmente por efecto de un cúmulo de ilusiones y expectativas infundadas, que incluso están dispuestos a perdonar a la pareja que los maltrata. Arce y Fariña (2006) los definen como estados psicológicos inadaptados con altos niveles de estrés, sentimientos de frustración, escasa confianza en sí mismos, bajo autoconcepto, sentimientos de desagrado y cólera; Hoyos et al. (2012) si es una dependencia patológica puede ser factor de riesgo para trastornos como la depresión, por lo que es necesario que se implementen programas de salud emocional en las instituciones educativas y universidades.

La dependencia y codependencia emocional en la literatura científica son vistas como trastornos de la personalidad por dependencia (Martín y De la Villa, 2019), trastornos psiquiátricos, relaciones de pareja patológica, enfermedades patológicas. El término de codependencia es colateral a la investigación, sin embargo, necesita ser definido de acuerdo a la evolución de la violencia interpersonal el que involucra una relación adictiva 
no solo con individuos, sino también con objetos o conductas. La persona codependiente siempre renuncia a sus intereses personales y la felicidad, entregando su vida a la persona dependiente, de lo contrario, puede sufrir de ansiedad, depresión, estrés o culpabilidad, a pesar de la ingratitud del dependiente (Sophia et al., 2007 y Castelló, 2005).

De la revisión de la literatura se recoge la necesidad de vigilar las adicciones de dependencia y codependencia, con la finalidad de promover organizar programas de alfabetización emocional para adolescentes y adultos, aprovechando la neuroplasticidad del cerebro e impulsar vínculos afectivos estables, equilibrados, fuertes y duraderos (Bornstein, et al. 2002 en Estévez, Urbiola, Iruarrizaga, Onaindia, y Jauregui, 2017) para que este grupo de personas practiquen una dependencia saludable o normal, con identidad fuerte, autoconcepto adecuado, confianza en los demás; sin tener miedo a quedarse solo o al abandono.

El objetivo general del estudio fue establecer la relación entre violencia y dependencia emocional en parejas adolescentes de educación secundaria de instituciones educativas públicas y privadas de la región Huancavelica, siendo los objetivos específicos determinar el nivel de violencia y el nivel de dependencia emocional. La hipótesis buscó determinar la relación entre violencia y dependencia emocional en parejas adolescentes de educación secundaria de la región Huancavelica.

\section{Metodología}

En la muestra seleccionada para el estudio se identificaron edades de 12 a 18 años, que corresponden a la adolescencia temprana, media y tardía, etapas de la vida humana vulnerable a la admisión de comportamientos de riesgo como las adicciones, la dependencia emocional y el inicio a la relación afectiva con la pareja. Esta etapa de desarrollo humano es calificado como adolescencia para el estudio, definición que se tomó de la OMS (2013a. parr. 1) “(...) el período de crecimiento y desarrollo humano que se produce después de la niñez y antes de la vida adulta, entre los 10 a 19 años".

Se seleccionó la muestra aplicando el instrumento y en ella un ítem: ¿tuviste pareja o enamorado en los últimos seis meses con una duración mínima de 30 días? Las encuestas con la respuesta "No" fueron excluidas de la muestra y las encuestas respondidas como "Sí" fueron incluidas a la muestra; alcanzando un total de encuestas validas de $n=205$ estudiantes adolescentes mujeres con pareja entre 12 y 18 años de edad. Los instrumentos se aplicaron en el mes de noviembre de 2019, en las siete provincias de la región Huancavelica-Perú, del segundo al quinto grado de secundaria.

\subsection{Instrumentos}

Se administró el Inventario para medir la violencia entre novios, de ocho dimensiones (desapego, humillación, sexual, coerción, física, género, castigo emocional e instrumental) y 42 ítems, validado por Rodríguez-Díaz et al. (2017). También se aplicó el cuestionario de cálculo dependencia emocional con cuatro dimensiones (ansiedad por separación, expresión afectiva de la pareja, modificación de planes y miedo a la soledad) de 23 ítems, validado por Lemos y Londoño (2006) que calculó el nivel de dependencia emocional en las adolescentes con pareja de educación secundaria encuestadas. Loscuestinarios solicitaban datos generales, como edad, procedencia y gestión de la institución educativa.

Los instrumentos fueron valorados con la escala de Likert, y luego se clasificaron en: a) Leve, con presencia fortuita de violencia o dependencia y controlable; b) Moderada, con signos, señales de violencia o dependencia, con exposición a riesgos, necesita ayuda; c) Severa, presencia frecuente de violencia o dependencia, la víctima y el perpetrador necesitan ayuda profesional urgente. 


\subsection{Procedimiento}

Para obtener los datos de las estudiantes se solicitó autorización a las instituciones educativas públicas y privadas a través de una carta. Asimismo, se presentó una declaración jurada de confidencialidad de la información. Aceptada la autorización se administraron los instrumentos en las instituciones educativas de las capitales de provincias y zonas accesibles de manera síncrona con una duración de 45 minutos.

\subsection{Análisis de datos}

El análisis de datos se realizó con el uso del programa estadístico SPSS (Statistical Package for the Social Sciences 25.0), para presentar las tablas de: medidas de tendencia central de la edad, frecuencia y porcentaje de las variables. La prueba de hipótesis se realizó con el coeficiente de correlación de la (Rho) de Spearman, por tener datos primigenios ordinales de ambos instrumnetos, con la finalidad de establecer la relación con un nivel de significancia de alfa ( $\alpha=5 \%)$.

\section{Resultados y discusión}

\subsection{Resultados}

En el presente estudio, se investigó la violencia y dependencia emocional en parejas adolescentes de educación secundaria de la región Huancavelica en el año 2019.

Cuadro 1

Edad de las estudiantes de educación secundaria

\begin{tabular}{|c|c|c|}
\hline \multicolumn{2}{|c|}{ Medidas estadítica } & edad \\
\hline \multicolumn{2}{|c|}{ Media } & 15,34 \\
\hline \multicolumn{2}{|c|}{ Mediana } & 15,00 \\
\hline \multicolumn{2}{|c|}{ Moda } & 15 \\
\hline \multicolumn{2}{|c|}{ Rango } & 6 \\
\hline \multicolumn{2}{|c|}{ Mínimo } & 12 \\
\hline \multicolumn{2}{|c|}{ Máximo } & 18 \\
\hline \multirow[t]{3}{*}{ Percentiles } & 25 & 15,00 \\
\hline & 50 & 15,00 \\
\hline & 75 & 16,00 \\
\hline
\end{tabular}

Fuente: Inventario de violencia en parejas adolescentes de educación secundarias

La edad mínima de la muestra encuestada fue 12 y la máxima 18 años, siendo la media y moda 15 años, donde se muestra que uno de cada dos estudiantes tuvo 15 años y el $75 \% 16$ años o menos.

En el cuadro 2 se observa que más del $76 \%$ de los estudiantes estuvieron cursando el cuarto y quinto grado, el $19 \%$ el tercer grado y solo el $4.9 \%$ el segundo grado. La mayoría de las estudiantes de educación secundaria que tienen pareja se encuentran cursando en el cuarto y quinto grado.

En la cuadro 3 se observa el $65.4 \%$ de estudiantes encuestados tuvieron lugar de procedencia de zona urbana y el $34.6 \%$ de zona rural, esto se debe que la mayoría de las instituciones de educación secundaria se encuentran en zona urbana. 
Cuadro 2

Grado escolar de parejas adolescentes de educación secundaria encuestadas

\begin{tabular}{ccc}
\hline & Frecuencia & Porcentaje \\
& $f_{i}$ & $p_{i}$ \\
\hline Segundo grado & 10 & 4,9 \\
Tercer grado & 39 & 19,0 \\
Cuarto grado & 79 & 38,5 \\
Quinto grado & 77 & 37,6 \\
Total & 205 & 100,0 \\
\hline
\end{tabular}

Fuente: Inventario de violencia en parejas adolescentes de educación secundarias

Cuadro 3

Procedencia de parejas adolescentes

de educación secundaria encuestadas

\begin{tabular}{ccc}
\hline & Frecuencia & Porcentaje \\
& $f_{i}$ & $p_{i}$ \\
\hline Zona urbana & 134 & 65,4 \\
Zona rural & 71 & 34,6 \\
Total & 205 & 100,0 \\
\hline
\end{tabular}

Fuente: Inventario de violencia en parejas adolescentes de educación secundarias

En el cuadro 4 se observa que más del $87 \%$ de las instituciones educativas de la región Huancavelica son de gestión pública y solo un $12,7 \%$ son de privada. Este resultado se debió que en la región Huancavelica se tiene un $97 \%$ de instituciones educativas de gestión pública y $3 \%$ de instituciones de gestión privadas.

\section{Cuadro 4}

Gestión administrativa de las instituciones educativas de las estudiantes encuestadas

\begin{tabular}{ccc}
\hline & Frecuencia & Porcentaje \\
& $f_{i}$ & $p_{i}$ \\
\hline Gestión pública de la IIEE & 179 & 87,3 \\
Gestión privada de la IIEE & 26 & 12,7 \\
Total & 205 & 100,0 \\
\hline Fuente: Inventario de violencia en parejas adolescentes de educación secundarias
\end{tabular}

Del cuadro 5 se concluye que más del $30 \%$ de estudiantes sufrieron violencia moderada y severa de parte de su parejas varones; es decir, dos de cada tres adolescentes mujeres de educación secundaria manifestaron haber sufrido un nivel de violencia leve de su pareja y una de cada tres adolescentes sufrieron violencia moderada y severa.

Cuadro 5

Frecuencia y porcentaje de nivel de violencia en parejas adolescentes de educación secundaria

\begin{tabular}{cccc}
\hline & Frecuencia & Porcentaje & Porcentaje acumulado \\
Nivel de violencia & $f_{i}$ & $p_{i}$ & $P$ \\
\hline Leve & 143 & 69,8 & 69,8 \\
Moderada & 52 & 25,3 & 95,1 \\
Severa & 10 & 4,9 & 100,0 \\
Total & 205 & 100,0 & \\
\hline
\end{tabular}

Fuente: Inventario de violencia en parejas adolescentes de educación secundarias 
Del cuadro 6 se concluye que más 33\% de estudiantes tienen tendencia a la dependencia emocional moderada y severa hacia sus parejas varones. Es decir, dos de tres estudiantes mujeres de educación secundaria con pareja tienen un nivel de dependencia leve o normal y uno de tres estudiantes tienen un nivel de dependencia moderada y severa, quienes necesitan ayuda profesional para una gestión emocional saludable.

\section{Cuadro 6}

Nivel de dependencia emocional de parejas adolescentes de educación secundaria de la región Huancavelica

\begin{tabular}{cccc}
\hline & Frecuencia & Porcentaje & Porcentaje acumulado \\
Nivel de dependencia emocional & $f_{i}$ & $p_{i}$ & $P$ \\
\hline Leve & 136 & 66,3 & 66,3 \\
Moderada & 61 & 29,8 & 96,1 \\
Severa & 8 & 3,9 & 100,0 \\
Total & 205 & 100,0 & \\
\hline
\end{tabular}

Fuente: Cuestionario de dependencia emocional en adolescentes de educación secundaria

En la contrastación de la hipótesis general se estableció el coeficiente de correlación con la Rho de Spearman de 0,730, que corresponde a una correlación positiva fuerte, esto explica que a mayor dependencia emocional de las estudiantes aceptan mayor violencia de la pareja. Asimismo, con una probabilidad de error de $0 \%$ existe una correlación directa y significativa entre violencia y dependencia emocional en estudiantes mujeres con pareja de educación secundaria, por lo que se acepta la hipótesis, tal como observamos en el cuadro 7.

\section{Cuadro 7}

Análisis de correlación de las variables violencia y dependencia emocional en parejas adolescentes de educación secundaria de la región Huancavelica

\begin{tabular}{|c|c|c|c|c|}
\hline & & & Violencia & Dependencia emocional \\
\hline Rho de & Violencia & Coeficiente de correlación & 1,000 &, $730^{* *}$ \\
\hline \multirow[t]{5}{*}{ Spearman } & & Sig. (bilateral) & . & ,000 \\
\hline & & $\mathrm{N}$ & 205 & 205 \\
\hline & Dependencia & Coeficiente de correlación &, $730^{* *}$ & 1,000 \\
\hline & emocional & Sig. (bilateral) & ,000 & . \\
\hline & & $\mathrm{N}$ & 205 & 205 \\
\hline
\end{tabular}

**. La correlación es significativa en el nivel 0,01 (bilateral).

Fuente: Inventario de violencia y cuestionario de dependencia emocional en parejas adolescentes de educación secundarias

En las pruebas de hipótesis específicas entre violencia y las dimensiones de dependencia emocional, se estableció el coeficiente de correlación con la Rho de Spearman entre 0,487 y 0,560, que corresponde a una correlación positiva débil y media, esto explica que a mayor violencia de sus parejas mayor dependencia emocional. Asimismo, con una probabilidad de error de $0 \%$ existe una correlación directa y significativa entre violencia y las dimensiones de dependencia emocional expuestas. 


\section{Cuadro 8}

Correlación de las variables específicas de violencia y dimensiones de dependencia emocional en parejas adolescentes de educación secundaria de la región Huancavelica

Correlaciones de violencia y ansiedad por separación en estudiantes mujeres con pareja de educación secundaria

\begin{tabular}{|c|c|c|c|}
\hline \multirow[t]{6}{*}{ Rho de Spearman } & Violencia en estudiantes & Coeficiente de correlación &, $553 * *$ \\
\hline & & Sig. (bilateral) & ,000 \\
\hline & & $\mathrm{N}$ & 205 \\
\hline & Ansiedad por separación & Coeficiente de correlación & 1,000 \\
\hline & & Sig. (bilateral) & . \\
\hline & & $\mathrm{N}$ & 205 \\
\hline \multicolumn{4}{|c|}{ Correlaciones de violencia y expresión afectiva en estudiantes mujeres con pareja de educación secundaria } \\
\hline \multirow[t]{6}{*}{ Rho de Spearman } & Violencia en estudiantes & Coeficiente de correlación &, $487 * *$ \\
\hline & & Sig. (bilateral) & ,000 \\
\hline & & $\mathrm{N}$ & 205 \\
\hline & Expresión afectiva de la pareja & Coeficiente de correlación & 1,000 \\
\hline & & Sig. (bilateral) & . \\
\hline & & $\mathrm{N}$ & 205 \\
\hline \multicolumn{4}{|c|}{ Correlaciones de violencia y modificación de planes en estudiantes mujeres de educación secundaria } \\
\hline \multirow[t]{6}{*}{ Rho de Spearman } & Violencia en estudiantes & Coeficiente de correlación &, $560 * *$ \\
\hline & & Sig. (bilateral) & ,000 \\
\hline & & $\mathrm{N}$ & 205 \\
\hline & Modificación de planes & Coeficiente de correlación & 1,000 \\
\hline & & Sig. (bilateral) & . \\
\hline & & $\mathrm{N}$ & 205 \\
\hline \multicolumn{4}{|c|}{ Correlaciones de violencia y miedo a la soledad en estudiantes mujeres de educación secundaria } \\
\hline \multirow[t]{6}{*}{ Rho de Spearman } & Violencia en estudiantes & $\begin{array}{c}\text { Coeficiente de } \\
\text { correlación }\end{array}$ &, $542 * *$ \\
\hline & & Sig. (bilateral) & ,000 \\
\hline & & $\mathrm{N}$ & 205 \\
\hline & Miedo a la soledad & $\begin{array}{l}\text { Coeficiente de } \\
\text { correlación }\end{array}$ & 1,000 \\
\hline & & Sig. (bilateral) & $\cdot$ \\
\hline & & $\mathrm{N}$ & 205 \\
\hline
\end{tabular}

**. La correlación es significativa en el nivel 0,01 (2 colas).

Fuente: Inventario de violencia y cuestionario de dependencia emocional en parejas adolescentes de educación secundarias

\subsection{Discusión}

En la investigación al establecer la relación entre violencia y dependencia emocional en parejas adolescentes de educación secundaria, se encontró que el coeficiente de correlación de la Rho de Spearman de 0,730, que corresponde a una correlación positiva fuerte. Esto explica que a mayor violencia hay mayor riesgo de dependencia emocional. A lo mencionado, con una probabilidad de error de $0 \%$ existe una correlación directa y significativa entre ambas variables. Resultados similares se encontraron en Rodríguez, Momeñe, Olave, Estévez y Iruarrizaga (2019) quienes en su investigación realizada en tres universidades españolas, explican la relación positiva entre la dependencia emocional y la violencia en las relaciones de pareja.

Otro estudio en adolescentes y adultos realizado en España por Martín y De la Villa (2019), también encontraron relación entre la dependencia emocional y el maltrato psicológico. Del mismo modo, Aiquipa (2015) indicó que existe relación estadísticamente significativa entre la variable dependencia emocional y violencia en parejas. 
La Universidad Autónoma del Estado de Hidalgo, México, Del Castillo Arreola, Hernández Díaz, Romero Palencia y Iglesias Hoyos (2015) explican en la discusión de resultados la relación positiva y estadísticamente significativa entre los factores de la violencia en el noviazgo y la dependencia emocional pasiva. Por otra parte, Gonzáles y Leal (2016), en la región Centro América, Panamá, encuentraron que la dependencia emocional es un factor de riesgo para ser víctima de violencia de pareja. Por su parte, Villa, García, Cuetos y Sirvent (2017) hallaron una relación estadísticamente significativa entre las variables dependencia emocional y la violencia de pareja.

Al analizar estos resultados y la información de la (OMS, 2013 b) se concluye que las mujeres en todo el mundo, en todos los estratos sociales y edades sufren violación a sus derechos universales establecidos por diferentes organismos internacionales, por parte de los hombres que imponen su supremacía y dominación como hecho establecido o normalizando en la sociedad. Esta vulneración a los derechos se da por la indiferencia de los Estados, la corrupción y el machismo de los órganos judiciales, asimismo el poco conocimiento de sus derechos por parte de las mujeres.

A través del Inventario de Violencia, se observa con preocupación que una de cada tres mujeres percibe un nivel de violencia moderado y severo de sus parejas varones, resultados similares se encontraron en estudios de la OMS (2017), se precisa que en el mundo un $30 \%$ de mujeres ha sufrido alguna forma de violencia de parte de su pareja en algún momento de su vida, afectando la salud física, emocional y económica de la víctima. Silva y Azeredo (2019), haciendo referencia a la OMS, dicen que la prevalencia mundial de violencia contra la mujer varía entre $29 \%$ y 62\%. Moral, et. al. (2017), citando a Jouriles et al. (2009), y Cienfuegos (2014), en Padilla y Cruz (2019) sostienen que las cifras de violencia pueden ser mayores y muchas de las estudiantes de educación secundaria están experimentando violencia sin saberlo.

Al determinar el nivel de dependencia emocional en parejas adolescentes de educación secundaria, los resultados reflejan que una de cada tres estudiantes tienen dependencia emocional moderada y severa hacia su parejas varones a pesar de que son violentos. Estos resultados son corroborados parcialmente por Gonzáles y Leal (2016) y Martín y De la Villa Moral (2019), porque en los estudios que presentan se observa mayor porcentaje de dependencia emocional en hombres y mujeres. El menor porcentaje de dependencia emocional en el estudio se debe a que las estudiantes de educación secundaria son adolescentes, es así que la violencia y la dependencia emocional lo estén percibiendo como algo intrascendente y que luego progresivamente admitirán con mucha tolerancia la subordinación y las conductas abusiva, por lo que necesitan ayuda profesional temprana para una gestión emocional saludable. Es necesario que las autoridades locales y regionales trabajen en desarrollar habilidades sociales saludables en niños y adolescentes para tener una sociedad con salud mental saludable.

\section{Conclusiones}

En la región de Huancavelica se demostró que uno de cada tres adolescentes con parejas de educación secundaria sufre violencia y son dependientes emocionales, y se estableció que existe una relación positiva y significativa entre las dos variables de investigación. Los adolescentes ejecutores, receptores de violencia y dependencia emocional necesitan programas continuos de aprendizaje de comportamientos saludables, esto está contemplado en el Currículo Nacional del (Minedu Perú, 2016) como parte de las competencias de los estudiantes de educación secundaria, sin embargo sus acciones son insuficientes, por lo que es necesario la intervención del Ministerio de Salud, Gobierno Local y Regional.

En la región Huancavelica hay un predominio del machismo en los hogares rurales y urbanos, por lo que es necesario continuar con investigaciones cualitativas para recoger información más profunda. La presencia de la violencia en las parejas de estudiantes de educación secundaria es el inicio o la aceptación de la violencia en sus 
futuras convivencia o matrimonios. Esto permite recomendar la necesidad de implementar programas de desarrollo de habilidades sociales que eviten la consecución de violencia en las mujeres.

Finalmente, es necesario realizar este tipo de investigación en otros departamentos o regiones con similares características. Además, se sugiere la gestión de habilidades en los adolescentes mujeres.

\section{Referencias bibliográficas}

Aiquipa Tello, J. (2015). Dependencia emocional en mujeres víctimas de violencia de pareja. Revista de Psicología, 33(2), 412-437. http://www.scielo.org.pe/pdf/psico/v33n2/a07v33n2.pdf

Arce, R., y Fariña, F. (2006). Galician programme for the treatment and re-education o convicted gender aggresors. Anuario de Psicología Jurídica, 16, 41-64.

https://www.redalyc.org/pdf/3150/315024763004.pdf

Bosch, J. M. (2009). La danza de las emociones: vives como sientes (EDAF S.L. (ed.)). https://books.google.com.pe/books?id=YzjuFjGS7VQC\&pg=PA95\&dq=jose+castello\&hl=es\&sa=X\&ved=0a hUKEwiezoKQuPfoAhWImuAKHXsfATUQ6AEISTAE\#v=onepage $\& \mathrm{q}=$ jose castello\&f=false

Castelló Blasco, J. (2005). Dependencia emocional: Características y tratamiento (A. Editorial (ed.)). Alianza Editoria. https://books.google.com.pe/books?id=CwqwoGqpq2UC\&dq=dependencia+emocional\&hl=es\&sa=X\&ved =0ahUKEwif7JrF4OjoAhWRA9QKHb1TB98Q6AEIMDAB

Castelló Blasco, J. (2013). La superación de la dependencia emocional (p. 190). Corona Borealis. ISBN 99781482724820. https://www.amazon.com/-/es/Jorge-Castello-Blasco/dp/1482724820

De la Villa Moral, María; García, Andrea; Cuetos; Glenda y Sirvent, C. (2017). Violencia en el noviazgo, dependencia emocional y autoestima en adolescentes y jóvenes españoles. Revista lberoamericana de Psicología y Salud, ISSN 2171-2069, Vol. 3, No. 1, 2012, Págs. 19-37, 8(1), 19-37. https://doi.org/10.23923/j.rips.2017.08.009

Defensoría Del Pueblo. (2018). Violencia Contra Las Mujeres. Perspectivas de las victimas, obstaculos e índices cuantitativos, 56. http://www.defensoria.gob.pe

Del Castillo Arreola, A., Hernández Díaz, M. E., Romero Palencia, A., y Iglesias Hoyos, S. (2015). Violencia en el noviazgo y su relación con la dependencia emocional pasiva en estudiantes universitarios. Psicumex, 5(1), 8-18. https://doi.org/10.36793/psicumex.v5i1.248

Erulkar, A. S. (2001). Experiencia de la coerción sexual entre los jóvenes de Kenya. 33-40. https://www.guttmacher.org/sites/default/files/article_files/3003304s.pdf

Estévez, A., Urbiola, I., Iruarrizaga, I., Onaindia, J., y Jauregui, P. (2017). Dependencia emocional en el noviazgo y consecuencias psicológicas del abuso de internet y móvil en jóvenes. Anales de Psicologia, 33(2), 260268. https://doi.org/10.6018/analesps.33.2.255111

García-Moreno, C., Guedes, A., y Knerr, W. (2013). Violencia infligida por la pareja. Organaización Paramericana de La Salud, 1-12. https://doi.org/WHO/RHR/12.365

García Fonseca, L. y Cerda de la O, B. (2012). Violencia de genero. Instituto Nacional de Psiquiatría Ramón De La Fuente M., 8. https://docplayer.es/23372841-Violencia-de-genero-dra-lourdes-garcia-fonseca-drabeatriz-cerda-de-la-o.html 
Gonzáles, H., y Leal, R. (2016). Dependencia Emocional Como Factor De Riesgo Para La Violencia De Pareja En Mujeres Del Sector Paraíso- Corregimiento Mateo Iturralde - Distrito De San Miguelito - Panamá . Emotional Dependency As a Risk Factor for Intimate Partner Violence in Women Del Se. Tendencias En Psicología, 1(2), 25-35. revistas.upagu.edu.pe/index.php/TP/article/download/332/256

Gracia, E. (2014). Intimate partner violence against women and victim-blaming attitudes among Europeans. Bulletin of the World Health Organization, 92(5), 380-381. https://doi.org/10.2471/BLT.13.131391

Hoyos, M. L., Jaramillo, C. J., Calle, A. M. G., León, Z. T. D., y De La Ossa, D. (2012). Perfil cognitivo de la dependencia emocional en estudiantes universitarios en Medellín, Colombia. Universitas Psychologica, 11(2), 395-404. https://doi.org/10.11144/javeriana.upsy11-2.pcde

Leen, E., Sorbring, E., Mawer, M., Holdsworth, E., Helsing, B., y Bowen, E. (2013). Prevalence, dynamic risk factors and the efficacy of primary interventions for adolescent dating violence: An international review. Aggression and Violent Behavior, 18(1), 159-174. https://doi.org/10.1016/j.avb.2012.11.015

Lemos Hoyos, Mariantonia y Londoño Arredondo, N. (2006). Design and Validation of the Emotional Dependence Questionnaire in Colombian Population. Acta Colombiana de Psicología, 9(2), 127-140.

Martín, B., y De la Villa Moral, M. (2019). Relación entre dependencia emocional y maltrato psicológico en forma de victimización y agresión en jóvenes. Revista Iberoamericana de Psicología y Salud, 10(2), 75. https://doi.org/10.23923/j.rips.2019.02.027

Minedu Perú. (2016). Curriculo nacional de la Educación Básica. Libro Currículo Nacional de La Educación Basica, 224. http://www.minedu.gob.pe/curriculo/pdf/curriculo-nacional-de-la-educacion-basica.pdf

Momeñe, J., Jáuregui, P., y Estévez, A. (2017). El papel predictor del abuso psicológico y la regulación emocional en la dependencia emocional. Behavioral Psychology/ Psicologia Conductual, 25(1), 65-78.

Moral, M. de la V., García, A., Cuetos, G., y Sirvent, C. (2017). Violencia en el noviazgo, dependencia emocional y autoestima en adolescentes y jóvenes españoles. Revista Iberoamericana de Psicologia y Salud, 8(2), 96107. https://doi.org/10.23923/j.rips.2017.08.009

NACIONES UNIDAS (PNUD). (2015). Objetivo 5: Lograr la igualdad entre los géneros y empoderar a todas las mujeres y las niñas. https://www.un.org/sustainabledevelopment/es/gender-equality/

OMS. (2003). Informe mundial sobre violencia y salud. Publicación Científica y Técnica No. 588, 1(588), 381. http://iris.paho.org/xmlui/bitstream/handle/123456789/725/9275315884.pdf?sequence=1\&isAllowed=y

Organización de las Naciones Unidas (ONU). (2014). Reino Unido: Relatora de la ONU pide más medidas para afrontar la violencia contra la mujer. https://news.un.org/es/story/2014/04/1299381

Organización Mundial de la Salud. (2013a). Desarrollo en la adolescencia. https://www.who.int/maternal_child_adolescent/topics/adolescence/dev/es/

Organización Mundial de la Salud. (2013b). Informe de la OMS destaca que la violencia contra la mujer es "un problema de salud global de proporciones epidémicas." Informe de La OMS Destaca Que La Violencia Contra La Mujer Es "Un Problema de Salud Global de Proporciones Epidémicas." https://www.who.int/mediacentre/news/releases/2013/violence_against_women_20130620/es/

Organización Mundial de la Salud. (2017). Violencia contra la mujer. Violencia Contra La Mujer. http://www.who.int/es/news-room/fact-sheets/detail/violence-against-women 
ORGANIZACIÓN MUNDIAL DE LA SALUD (OMS). (2015). Estrategia Mundial para la Salud de la Mujer, el Niño y el Adolescente (2016-2030). Organizacion Mundial de La Salud, 108.

http://www.who.int/maternal_child_adolescent/documents/women-deliver-global-strategy/es/

Ospina-Ramírez, D. A., y Mosquera Ñañez, J. M. (2019). Rastros de violencia institucional: retos para el gerente educativo como constructor de paz en la escuela. Revista Colombiana de Educación, 1(79), 203-222. https://doi.org/10.17227/rce.num79-7504

Padilla Gámez, Nélida y Cruz del Castillo, C. (2019). Salud emocional y sexual: Una aproximación para el autocuidado y empoderamiento femenino (U. Iberoamericana (ed.)).

https://books.google.com.pe/books?id=jJGqDwAAQBAJ\&printsec=frontcover\&dq=La+salud+emocional\&hl =es\&sa=X\&ved=0ahUKEwjw-8SJ0-noAhUcGbkGHVibCWcQ6AEIbzAJ\#v=onepage\&q=La salud emocional\&f=false

Pérez, J.G.R.; Momeñe, J.; Olave, L.; Estévez, A. y Iruarrizaga, I. (2019). La dependencia emocional y la resolución de conflictos en heterosexuales, homosexuales y bisexuales (pp. 59-75). https://www.aesed.com/upload/files/v44n1_art4.pdf

Programa de las Naciones Unidas para el Desarrollo (PNUD). (2000). Objetivos de Desarrollo del Milenio. https://www.undp.org/content/undp/es/home/sdgoverview/mdg_goals.html

Racine, R. H. y Zeytinoglu, S. (2012). African Americans and Teen Dating Violence. 40(1), 20-32. https://doi.org/10.1080/01926187.2011.578033

Rodríguez-Díaz, F. J., Herrero, J., Rodríguez-Franco, L., Bringas-Molleda, C., Paíno-Quesada, S. G., y Pérez, B. (2017). Validation of Dating Violence Questionnarie-R (DVQ-R). International Journal of Clinical and Health Psychology, 17(1), 77-84. https://doi.org/10.1016/j.ijchp.2016.09.001

Rodríguez, J. G.; Momeñe, J.; Olave, L.; Estévez, A. y Iruarrizaga, I. (2019). La dependencia emocional y la resolución de conflictos en heterosexuales, homosexuales y bisexuales. 44(1), 59-75.

https://www.aesed.com/upload/files/v44n1_art4.pdf

Roque Latorre, P. (2015). Salud Integral del Adolescente 3. Grupo Editorial Patria.

https://books.google.com.pe/books?id=L81UCwAAQBAJ\&printsec=frontcover\&hl=es\&source=gbs_ge_su mmary_r\&cad $=0 \# v=0$ nepage $\& q=$ noviazgo $\& f=$ false

Ruiz-pérez, I., Escribà-agüir, V., y Montero-pi, I. (2017). Atención Primaria Prevalence of intimate partner violence in Spain : A national cross-sectional survey in primary care. 49(2).

Sherer, M. (2009). The nature and correlates of dating violence among Jewish and Arab youths in Israel. Journal of Family Violence, 24(1), 11-26. https://doi.org/10.1007/s10896-008-9201-8

Silva, A. N., y Azeredo, C. M. (2019). Associação entre vitimização por violência entre parceiros íntimos e depressão em adultos brasileiros. Ciencia \& Saude Coletiva, 24(7), 2691-2700. https://doi.org/10.1590/1413-81232018247.25002017

Sophia, E. C., Tavares, H., y Zilberman, M. L. (2007). Pathological love: Is it a new psychiatric disorder? Revista Brasileira de Psiquiatria, 29(1), 55-62. https://doi.org/10.1590/S1516-44462007000100016

Vagi, K. J., Olsen, E. O. M., Basile, K. C., y Vivolo-Kantor, A. M. (2015). Teen dating violence (physical and sexual) among US high school students: Findings from the 2013 National Youth Risk Behavior Survey. JAMA Pediatrics, 169(5), 474-482. https://doi.org/10.1001/jamapediatrics.2014.3577 
Vallejo, A. (2019). Dependencia Emocional.

https://books.google.com.pe/books?id=CzKyDwAAQBAJ\&printsec=frontcover\&dq=dependencia+emocion al\&hl=es\&sa=X\&ved=0ahUKEwif7JrF4OjoAhWRA9QKHb1TB98Q6AEIJzAA\#v=onepage\&q=dependencia emocional\&f=false

Villegas Moreno, M. J., y Sanchez Osorio, N. (2013). Dependencia emocional en un grupo de mujeres denunciantes de maltrato de pareja. Textos y Sentidos, 7(1), 20.

http://biblioteca.ucp.edu.co/OJS/index.php/textosysentidos/article/view/807

Zamora-Damián, G., Alvídrez Villegas, S., Aizpitarte, A., y Rojas-Solís, J. L. (2018). Prevalencia De Violencia En El Noviazgo En Una Muestra De Varones Adolescentes Mexicanos. Revista de Psicología y Ciencias Del Comportamiento de La Unidad Académica de Ciencias Jurídicas y Sociales, 9(1), 30-53. https://doi.org/10.29365/rpcc.20180529-63

Esta obra está bajo una Licencia Creative Commons Attribución-NoCommercial 4.0 International

(cc) EY-NC 